\title{
Early Hepatocellular Carcinoma: Diagnosing the Difficult Nodule
}

\author{
Saleem Farooqui ${ }^{1}$, Natarajan Ravendhran ${ }^{2}$, Steven C. Cunningham ${ }^{3 *}$ \\ ${ }^{1}$ Department of Radiology, Saint Agnes Hospital, Baltimore, USA; ${ }^{2}$ Department of Medicine, Saint Agnes Hospital, Baltimore, USA; \\ ${ }^{3}$ Department of Surgery, Saint Agnes Hospital, Baltimore, USA. \\ Email: ${ }^{*}$ steven.cunningham@stagnes.org
}

Received January $21^{\text {st }}, 2013$; revised February $23^{\text {rd }}, 2013$; accepted March $3^{\text {rd }}, 2013$

Copyright (C) 2013 Saleem Farooqui et al. This is an open access article distributed under the Creative Commons Attribution License, which permits unrestricted use, distribution, and reproduction in any medium, provided the original work is properly cited.

\begin{abstract}
The incidence of hepatocellular carcinoma (HCC) is rising worldwide. Although the best chance for long-term survival is early detection, screening high-risk populations to detect HCC when it is most treatable still has only limited success. Once detected within the cirrhotic liver, many observations still defy correct characterization, due in part to a history of nonstandarized nomenclature and reporting patterns. Recently, however, an initiative by the American College of Radiology, Liver Imaging-Reporting and Data System (LI-RADS), has begun to remedy these inadequacies. Here, we review LI-RADS, and focus in particular on the difficult nodule, i.e., a radiological observation that challenges our current diagnostic ability, and review essential technical imaging features that aid in the diagnosis of early HCC.
\end{abstract}

Keywords: HCC; Hepatocellar; Carcinoma; Dysplasia; Nodule; LI-RADS; Liver Imaging-Reporting and Data System; MRI; CT; Liver Imaging; Screening

\section{Introduction}

The incidence of hepatocellular carcinoma (HCC) is increasing globally and is the sixth most common cancer worldwide [1-3]. The incidence is highest among men, at $7.9 \%$ of all the cancers, with a mortality rate of $11.3 \%$ (3.7\% and $2.2 \%$, respectively, for women) [1]. In the USA, 26,190 new cases were diagnosed in 2011 and 19,590 deaths are due to HCC [4].

In Asian Countries and in African countries the rise is predominantly secondary to Hepatitis B infection, whereas in USA and Japan, it is due to the Hepatitis $\mathrm{C}$ infection. It has been observed and projected to rise among the patients suffering from NASH and metabolic syndrome [5]. Systematic review of the literature has revealed a positive correlation between diabetes and the increased risk of HCC in Chinese cohorts [6]. The other risk factors are alcoholic cirrhosis, hemochromatosis, alpha-1 antitrypsin deficiency and patients with autoimmune hepatitis induced cirrhosis.

\section{Screening}

While some level-1 (randomized controlled trial [RCT])

${ }^{*}$ Corresponding author. evidence suggests that screening for hepatocellular carcinoma in high-risk patients may improve survival [7], a more recent Cochrane analysis including three RCTs concluded that there is insufficient evidence to either support or refute the value of screening even high-risk hepatitis-B-positive patients, using alpha-fetoprotein (AFP) and ultrasound (US) screening [8]. A RCT by Trinchett, et al., comparing 3- and 6-month periodicities of US screening showed that at the time of initial screening diagnosis, $10 \%$ of patients already have infiltrative disease, $11 \%$ have vascular involvement, and $25 \%$ have tumor burden beyond Milan criteria [9].

The current screening tools that are widely used include liver US and the measurement of serum AFP, generally every 6 months in high risk patients. However, despite these guidelines, the resectable (potentially curable) lesions are identified infrequently due to the limitations of these methods. When used for screening highrisk individuals, US has sensitivity of only $65 \%-80 \%$ but a specificity greater than $90 \%$ [10]. Limitations of US include the interoperator variability, limitations of body habits, such as the severe obesity that is epidemic in the United States, and the lack of contrast-enhanced US in the United States. Although AFP has relatively poor sensitivity and specificity, new markers are on the hori- 
zon $[11,12]$.

\section{Liver Imaging-Reporting and Data System (LI-RADS)}

Once a lesion is detected, either as a result of screening or of evaluation of symptoms, it is essential that it be completely and correctly described, not only for immediate diagnosis of the difficult nodule for a given patient, but also for standardizing reporting among different patients, for improving communication among clinicians, for performance auditing and quality assurance, and for research endeavors. To this end, the American College of Radiology has developed the Liver Imaging-Reporting and Data System (LI-RADS) for the diagnosis of HCC, recently reviewed in a case-based format by Purysko et al. [13]. Akin to the widely used BI-RADS acronym (Breast Imaging-Reporting and Data System), LI-RADS aims to reduce variability in the interpretation of liverimaging studies in patient with cirrhosis or otherwise at risk for the development of HCC [14].

The need for standardized reporting of liver lesions using LI-RADS is amplified by the increasing sophistication of the technology, both in computed tomography (CT) and magnetic resonance imaging (MRI) $[15,16]$, with a corresponding increased need for subspecialization [17] among radiologists. Axial imaging technology and the degree of subspecialized skill of those interpreting the resulting images have increased so much over the past 2 decades as to largely supplant biopsy for classically enhancing lesions. In fact, the rate of histologically unconfirmed HCC has increased nearly threefold faster than the rate of biopsy-confirmed HCC [18], due to recent increases in imaging-base diagnosis. Accordingly, the National Comprehensive Cancer Network (NCCN) guidelines [19] recommends that for lesions $>1 \mathrm{~cm}$, when two classical enhancements are seen, the diagnosis of HCC is made without need for biopsy, where classic enhancement is considered to be arterial hyperenhancement and venous washout. In addition to these two tumor characteristics, an increased diameter $\geq 1 \mathrm{~cm}$ within 1 year, and tumor within the lumen of a vein are the most concerning characteristics of a liver mass [13] (Figure 1).

Analogous to the improvements on the horizon regarding the standardization of operative reporting, using synoptic electronic reporting (as opposed to traditional dictated narrative reporting), with consequent benefits such as more complete, more usable, more accurate information [20-22], the structured reporting produced by LI-RADS similarly may be expected to effectively address recognized deficiencies [23] in current imaging and reporting techniques, with certain improvement in patient care and research activities.

Building on the structure of the well established BI-
RADS system, a complete LI-RADS evaluation assigns an imaging observation (e.g., cirrhosis-associated liver nodule) to one of five categories (Table 1 and Figure 2), ranging from definitely benign (LI-RADS 1) to definitely HCC (LI-RADS 5, Figure 3), with intermediate categories being LI-RADS 2 (probably benign, Figure 4), LIRADS 3 (intermediate probability, Figure 5), and LIRADS 4 (probably HCC) [14]. Although the lesion under evaluation may be hypo- or hyperintense on the precontrast MRI, the essential hyperintensity during the arterial phase may still occur and must be followed by venous washout to hypointensity relative to the surrounding liver parenchyma; similarly hypointensity on the venous phase is insufficient for the diagnosis of HCC in the absence of arterial hyperintensity (Figure 6).

While one may envision a future LI-RADS system usable not only for the initial CT or MRI diagnosis but for surveillance imaging following resection or nonresectional liver-directed therapy, the current version of LIRADS applies to only untreated observations in patients at elevated risk for HCC, and does not incorporate contrast-enhanced US [14].

\section{The Difficult Nodule}

Cirrhosis-associated hepatocellular nodules arising in response to liver injury may be difficult to diagnosis, especially when small. The differential diagnosis includes regenerative nodules, dysplastic nodules, siderotic nodules (which may be either regenerative or dysplastic histologically), as well as "non-nodule nodules", such as arterial enhancement within cirrhotic liver due to arterialportal shunts, transient hepatic attenuation/intensity difference, and rarely, hemangiomas such that the diagnostic and therapeutic management of such difficult nodules (observations) is challenging.

\subsection{Regenerative Nodules and Dysplastic Nodules}

A regenerative nodule is a well-defined region of parenchyma that has enlarged in response to necrosis, altered circulation, or other stimuli; they are present in all cirrhotic livers and are surrounded by fibrous septae. Regenerative nodules may be classified according to size as either micronodules $(<3 \mathrm{~mm})$ or macronodules $(\geq 3 \mathrm{~mm})$. Rarely, so-called "giant" regenerative nodules have also been described as measuring up to $5 \mathrm{~cm}$ [24]. Although regenerative nodules greater than $1.5 \mathrm{~cm}$ have an increased likelihood of harboring dysplastic or malignant foci [25], nodules measuring $2 \mathrm{~cm}$ have been observed in patients with Budd-Chiari syndrome [26] and autoimmune hepatitis [27]. Unlike cirrhosis, regenerative nodules in Budd-Chiari syndrome are not surrounded by fibrosis [24]. 
Table 1. Imaging features by LI-RADS category.

\begin{tabular}{|c|c|c|}
\hline LI-RADS Category & Description & Imaging Features \\
\hline 1 & Definitely benign & Diagnostic for a benign entity, or definite disappearance without treatment \\
\hline 2 & Probably benign & $\begin{array}{c}\text { Suggestive of a benign entity; or } \\
\text { Stable imaging features for } \geq 2 \mathrm{y} \text {; or } \\
\text { Probable disappearance in the absence of treatment }\end{array}$ \\
\hline 3 & $\begin{array}{l}\text { Intermediate } \\
\text { probability of } \\
\text { being HCC }\end{array}$ & $\begin{array}{l}\quad \leq 2 \mathrm{~cm} \text { : } \\
\text { Masslike configuration with arterial-phase hyperenhancement and no additional major features }{ }^{1} \text {, or } \\
\text { Masslike configuration with arterial phase hypoenhancement and } \leq 1 \text { additional major feature }{ }^{1} \\
\underline{\geq 2 \mathrm{~cm} \text { : }} \\
\text { Masslike configuration with arterial phase hypoenhancement and no additional major features }{ }^{1} \\
\text { Any size: } \\
\text { Nonmasslike configuration and neither LR-1 nor LR-2; or } \\
\text { Cannot be categorized as LR-1, LR-2, LR-4, or LR-5; or } \\
\text { Meets criteria for LR-4 or LR-5, with stablity for } \geq 2 \text { y }\end{array}$ \\
\hline 4 & Probably HCC & $\begin{array}{l}\text { Category A }(<2 \mathrm{~cm}) \text { : } \\
\text { Masslike configuration with arterial phase hyperenhancement and } 1 \text { additional major feature }{ }^{1} \text {, or } \\
\text { Masslike configuration with arterial phase iso- or hypoenhancement and } 2 \text { additional major features }{ }^{1} \text {, or } \\
\text { Probable tumor within lumen of vein } \\
\text { Category B }(\geq 2 \mathrm{~cm}):\end{array}$ \\
\hline 5 & Definitely HCC & $\begin{array}{l}\text { Category } \mathrm{A}(\geq 1 \mathrm{~cm} \text { but }<2 \mathrm{~cm}) \text { : } \\
\text { Masslike configuration with arterial phase hyperenhancement and } 2 \text { additional major features }{ }^{1} \text {, or } \\
\text { Definite tumor within lumen of vein } \\
\text { Category } \mathrm{B}(\geq 2 \mathrm{~cm}):\end{array}$ \\
\hline
\end{tabular}

Adapted from References [13,14]; ${ }^{1}$ Additional major features: portal venous phase or later phase hypoenhancement, increase in diameter of at least $1 \mathrm{~cm}$ within 1 year.

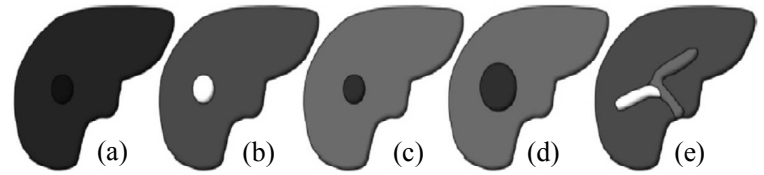

Figure 1. Schematic of imaging features characteristics of HCC used by LI-RADS, (a) Masslike configuration; (b) Arterial-phase hyperenhancement; (c) Portal venous phase hypoenhancement; (d) Increase of $\geq 1 \mathrm{~cm}$ in diameter within 1 year; (e) Tumor within the lumen of a vein. Adapted from Purysko, et al. [13].

Siderotic nodules, although reliably detected at MRI (Figure 7), are associated with no imaging criteria to reliably distinguish between regenerative or dysplastic histology [28]. Furthermore, siderotic nodules have not shown an increased propensity to develop into HCC, and the iron deposits may represent a marker for hepatic disease activity rather than a step in hepatocarcinogenesis [29].

Dysplastic nodules are found in $15 \%-25 \%$ of cirrhotic livers [30], and are stratified as low-grade or highgrade. Dysplastic nodules may occasionally be larger than $15 \mathrm{~mm}$ but rarely exceed $20 \mathrm{~mm}$ [31]. While low- grade dysplastic nodules resemble regenerative nodules histologically and are considered to have little potential for progression to HCC [32,33], high-grade dysplastic nodules are considered premalignant with more frequent and rapid progression to HCC, and are akin to the previously reported entity "atypical adenomatous hyperplasia" $[32,34]$. High-grade dysplastic nodules are characterized by both cytologic and architectural atypia and are difficult to distinguish even pathologically from well-differentiated HCC [35], although an emerging group of markers has proven quite accurate (HSP70, glypican 3 and glutamine synthetase) [36,37].

Not only is it difficult for a given pathologist or radiologist to distinguish between high-grade dysplasia and early HCC, but there is lack of consensus in histologic characterization between Western and Eastern pathologists regarding borderline lesions: What Western pathologists may call high-grade dysplasia, Easterners tend to interpret as early, well-differentiated HCC [38,39], although consensus is being reached recently [37]. Prior to consensus, patients with high-grade dysplastic nodules have been thought to be at fourfold higher risk of developing HCC [32], although many of the early HCCs di- 


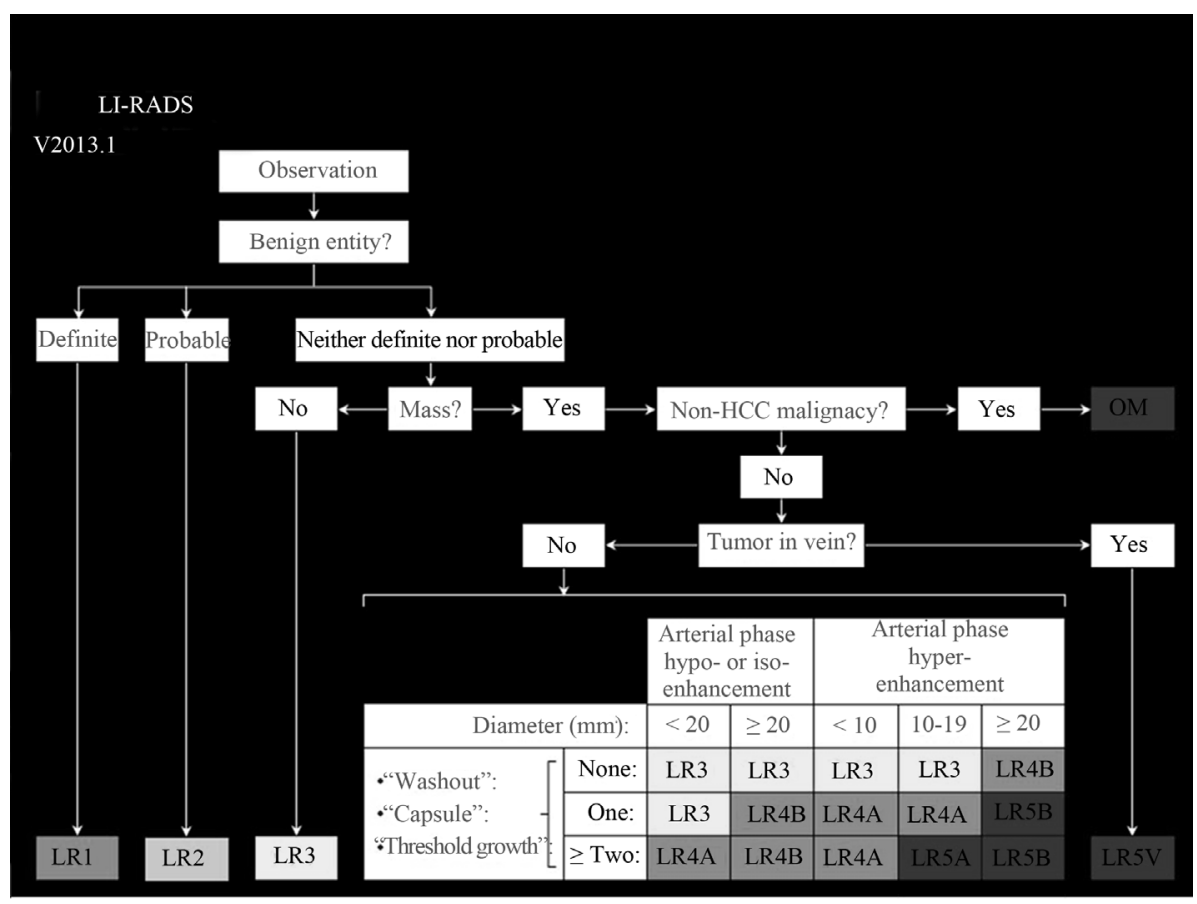

Figure 2. LI-RADS overview, Abbreviations: OM, other malignancy. See Table 1 for explanations of categories. Adapted from American College of Radiology, Reference [14].

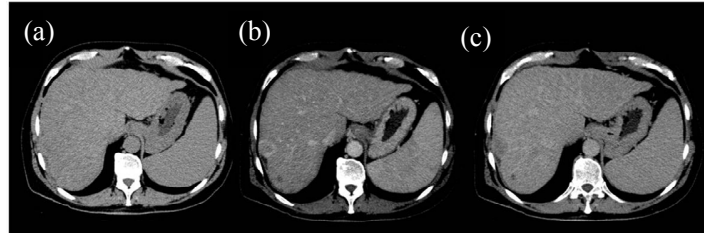

Figure 3. LI-RADS 5: Definitely HCC. A 2.8-cm hepatic lesion in segment 7/8: (a) Isodense on precontrast phase; (b) Demonstrating arterial-phase hyperenhancement; and (c) Venous phase hypoenhancement relative to surrounding liver.

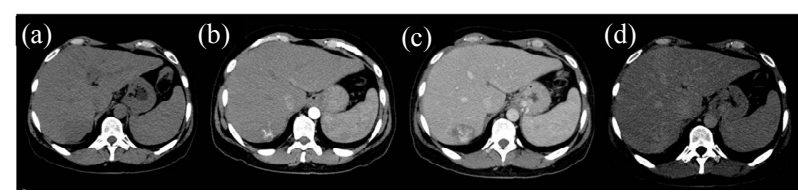

Figure 4. LI-RADS 2: Probably benign. Lesion in hepatic segment 7: (a) Isodense on precontrast phase; (b) and (c) Arterial-phase nodular hyperenhancement; (d) Demonstrating delayed venous return to isoenhancement.

agnosed in Japan tend to be reported as dysplastic nodules by Western pathologists [39]. According to the latest guidelines from the American Association of the Study of Liver Diseases, dysplastic nodules should not be treated or managed as cancers, and patients with known or suspected dysplastic nodules should be surveilled at 3- to 6-mo intervals [35].

A strong correlation exists between the perfusion source of hepatocellular nodules and the grade of malig-

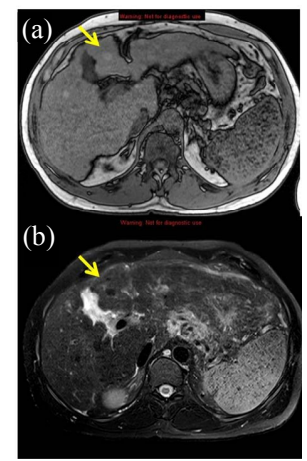

Figure 5. LI-RADS 3: Intermediate probability of being HCC. Opposed-phase T1 (a) and T2-fat saturated (b) images demonstrating T1-hyperintense nodules (arrows) with T2 hypointensity within a cirrhotic liver segment 4; the lesion did not enhance and is probably a dysplastic nodule.

nancy; that is, the intranodular portal supply relative to the surrounding liver decreases, whereas the arterial supply increases in accordance with elevation of the grade of malignancy of the nodule [34]. A major shift in angiogenesis typically occurs during the transition from lowgrade to high-grade dyplasia, and as little as $6 \%$ of $\mathrm{HCC}$ demonstrate residual portal blood supply whereas $94 \%$ show greater arterial supply compared to surrounding liver parenchyma [40]. Histologically, there are two related yet separate processes which account for the increased arterial supply of nodules from dysplasia to malignancy within the cirrhotic liver: (a) the hepatic sinusoids undergo gradual changes to resemble more ordi- 


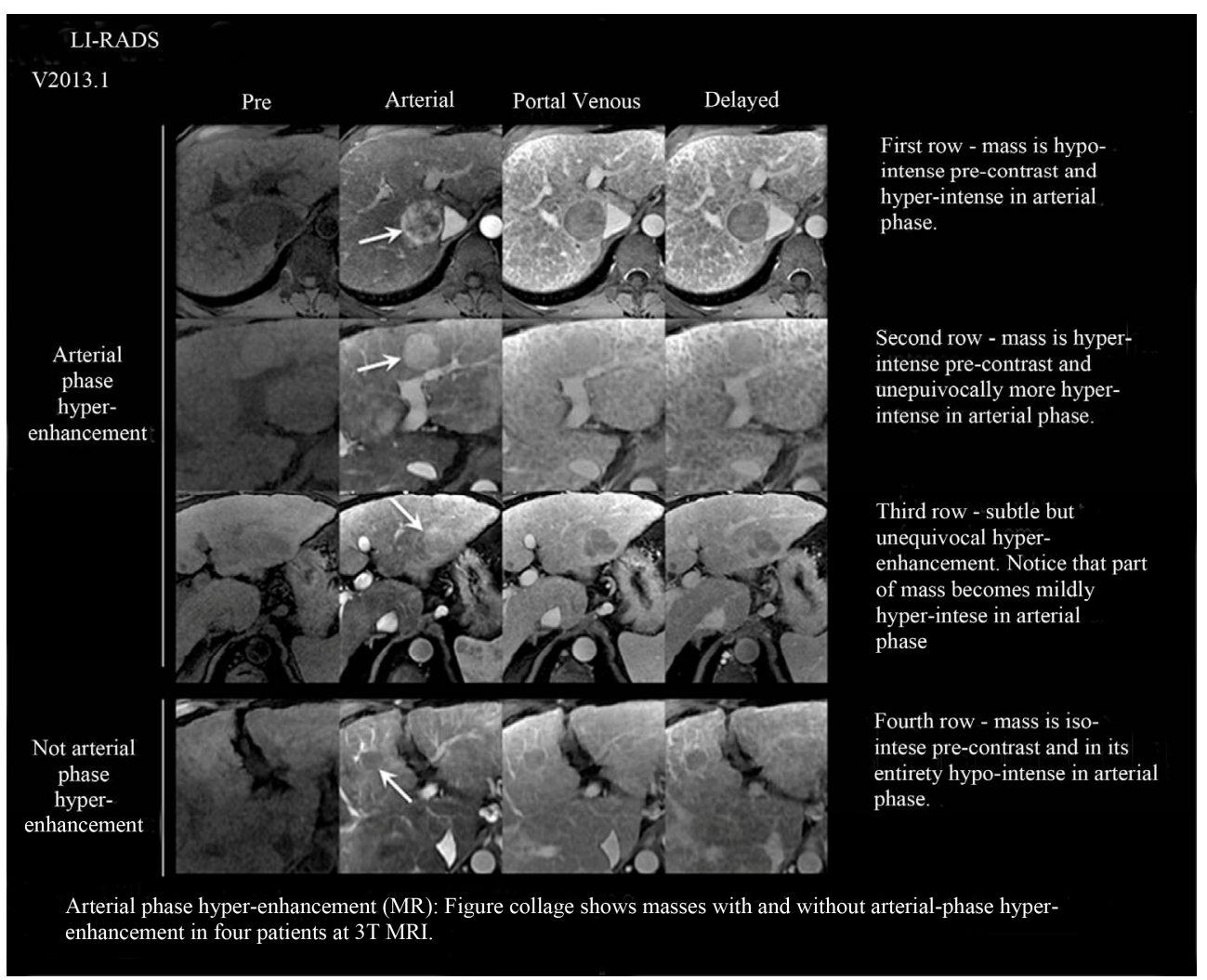

Figure 6. Classic arterial-phase hyperenhancement and venous-phase washout on MRI. See text for explanation. Adapted from American College of Radiology, Reference [14].

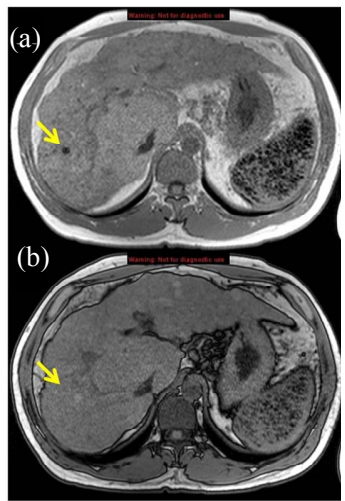

Figure 7. Siderotic nodule on MRI, dual-echo gradient recalled echo T1 images: (a) in-phase; (b) opposed-phase demonstrating susceptibility artifact classically associated with siderotic nodule (arrows). Also note, iron-containing GamnaGandy bodies in spleen.

nary capillaries, and (b) induction of new, unpaired arteries (neoangiogenesis) [31].

\subsection{Other "Nodules"}

There are several causes of nontumoral arterial enhancement within the cirrhotic liver that are important to distinguish, most of which are related to arterial-portal shunts, transient hepatic attenuation or intensity differences as occur with perfusion alteration (Figure 8), and rarely, hemangiomas $[41,42]$. Shunts are commonly peripheral or wedge-shaped but also can be nodular or irregular, and do not displace internal vasculature $[43,44]$. The underlying inflammatory-regenerative process of cirrhosis obliterates typical hemangiomas and, therefore, the residual observed hemangiomas often have atypical imaging features [45]. Other enhancing lesions found in the noncirrhotic liver (focal nodular hyperplasia, hepatic adenomas, hypervascular metastases, intrahepatic cholangiocarcinoma) are beyond the scope of this review, but can lead to diagnostic uncertainty when evaluating for $\mathrm{HCC}$; correlation with additional clinical information is essential.

\subsection{The Small Nodule}

The detection of small tumors remains the most challenging area in imaging a cirrhotic liver at risk for developing HCC. Although MRI reliably outperforms CT in evaluation for HCC [46-48], size remains a limitation for accurate characterization. The use of US in this role, although described, has been seriously questioned [46, 49], especially given the current unavailability of contrast-enhanced US in the United States. The technological advances leading to improved spatial, temporal, and contrast resolution of CT and MRI make them promising 


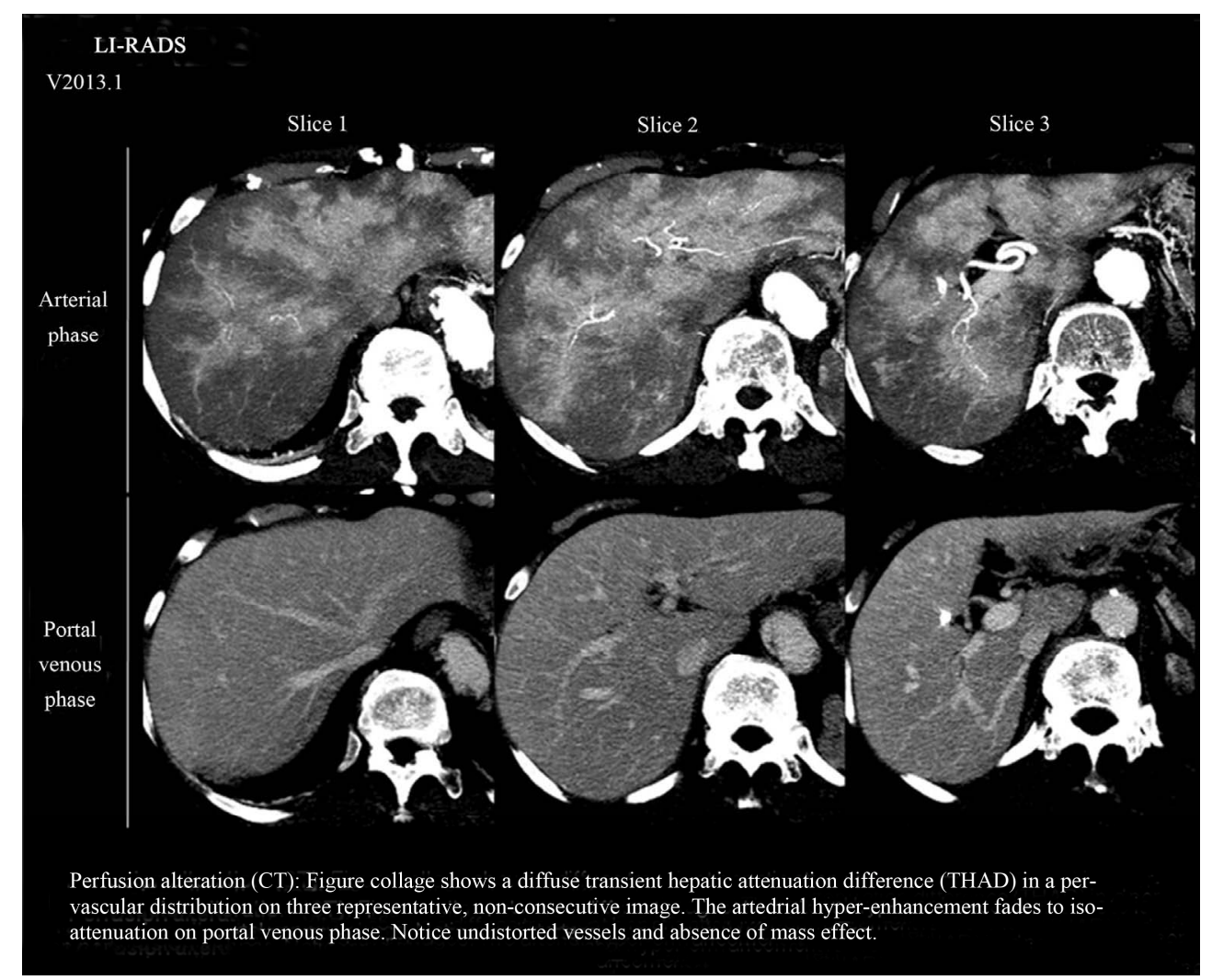

Figure 8. Perfusion alteration ("Nonnodules"), the areas of hyper-enhancement fail to meet the definition of a mass (3-dimentional, space-occupying lesion that displaces or replaced underlying hepatic parenchyma. Adapted from American College of Radiology, Reference [14].

alternatives to US in diagnosis and surveillance of HCC.

Several studies have suggested that the majority of arterial enhancing lesions $<2 \mathrm{~cm}$ in the cirrhotic liver are benign, even in patients with proven $\mathrm{HCC}$ elsewhere in the liver [41,50,51]. Yet, it is increasingly clear that such arterial-enhancing lesions should not be completely ignored, nor necessarily characterized as false-positive results, as many of these lesions likely represent premalignant dysplastic nodules or early (well-differentiated) HCC $[31,52,53]$. In addition, there are known limitations and pitfalls related to image-guided biopsy of small nodules in particular, with increased technical difficulty $[31,54]$ low yield [55], and false-negative results [56]. Arterialenhancing foci shown on MRI should at least be monitored closely [54]. Delayed hypointensity of an arteriallyenhancing lesion is an important feature that increases the specificity of diagnosing small $\mathrm{HCC}(<2 \mathrm{~cm})$, although its absence certainly does not exclude malignancy, since some early malignancies and high-grade dysplastic nodules may have residual portal perfusion rendering them isodense (isointense) on delayed phase imaging [33,42,57-59].

A report from Choi, et al. [60] indicated that retrospectively discovered small, indeterminate hepatocellular nodules $<2 \mathrm{~cm}$ in size, many of which were seen as foci of arterial enhancement only, did not become untreatable HCC despite a delay in diagnosis of up to 12 months, suggesting that this subset may represent a less aggressive pattern of HCC. Therefore, the need for very shortterm follow-up ( $<6$ months) of small, indeterminate nodules is questionable. As the size of the lesion (and likelyhood for malignancy) increases beyond $1 \mathrm{~cm}$, and fewer tumor doublings are required to reach a stage of poorer prognosis, shorter follow-up intervals may be of more benefit $[60,61]$. Prior reports have shown that nonneoplastic, arterial hypervascular lesions are the major cause of false-positive diagnoses of lesions smaller than $1 \mathrm{~cm}$, and dysplastic nodules are the major cause for lesions 1 $2 \mathrm{~cm}[62,63]$.

The issue of utmost importance and relevance surrounding the diagnostic challenges of small hepatocellular nodules is that of resource (viz. organ) allocation. False-positive diagnoses result in unfair priority granted to patients without malignancy, or even transplantation in patients in the absence of HCC [64]. Confident diagnosis of cancer is, therefore, a critical step prior to treatment indication and eventual organ allocation. It is precisely for this reason that both the American Association for the Study of Liver Diseases and the European Association for the Study of the Liver require concordant findings 
typical of HCC (e.g. both arterial phase enhancement and portal/delayed phase washout) on two imaging modalities when establishing the diagnosis for nodules $<2 \mathrm{~cm}$ in size; doing so essentially raises the specificity for malignancy near $100 \%$ [65].

\subsection{Technical Considerations Regarding Imaging (MRI) of HCC and Related Lesions}

In comparison to $\mathrm{CT}$, MRI has several advantages, including superior contrast resolution, the availability of variety of liver-specific contrast agents, making it likely the most important modality for the assessment of cirrhosis-associated hepatocellular nodules. The foundation of MRI assessment of the cirrhotic liver includes T1 gradient-echo, opposed phase sequences, high-quality, breathhold T2-weighted imaging with fat saturation, and volumetric T1-weighted gradient-echo imaging with fat saturation before and after bolus-infused gadolinium contrast during the arterial, portal, and equilibrium phases. Diffusion-weighted sequences are used at some institutions to evaluate hepatocellular nodules, and have been observed to have equal or better detection and characterization rates for HCC in comparison to standard breath-hold T2-weighted imaging $[66,67]$.

The ability of MRI to differentiate between lesions derives in part from super paramagnetic iron oxide (SPIO) particles, which are phagocytized by Kupfer cells in the liver (and spleen). In the cirrhotic liver, they accumulate in regenerative nodules, some dysplastic nodules, and regions of surrounding liver parenchyma, causing signal loss of T2 and T2* weighted sequences. Most HCC lesions lack Kupfer cells and, as a result, do not take up the SPIO particles, rendering these lesions hyperintense relative to the surrounding parenchyma $[25,68]$. Their conspicuity is dependent on the difference in the number of Kupfer cells within the nodules and the surrounding cirrhotic parenchyma, and certain well-differentiated HCC (containing Kupfer cells) can be distinguished from poorlydifferentiated malignancies [68].

The addition of a gadolinium-based contrast agents with specificity for hepatocellular uptake and biliary excretion (Eovist $^{(B}$ or Primovist ${ }^{\circledR}$, and Multihance $\left.{ }^{(}\right)$allows for further delayed imaging and enhancement of functioning hepatocytes, increasing the diagnostic accuracy for small liver lesions when compared to standard CT and MRI [6971]. In particular, this class of MR contrast agents assists in differentiation of small HCC with arterial-portal shunts, a known source of false-positive results on CT and conventional MRI [70-72]. The use of liver-specific contrast media for the identification of atypical lesions is already incorporated in the Japanese consensus-based clinical practice guidelines [73].

The image quality of the delayed (hepatocellular) phase is reduced in end-stage cirrhosis primarily due to poor hepatocellular function, resulting in decreased and delayed concentration of the gadolinium chelate within the hepatocytes and poor biliary concentration, reducing lesion-to-liver contrast-to-noise ratio $[63,71]$.

\subsubsection{Regenerative Nodules}

The T1 signal intensity of regenerative nodules is variable but usually indistinct; lipid-containing regenerative nodules show signal loss on opposed-phase gradient-echo sequences. A single lipid-containing nodule is suggestive of a dysplastic or malignant process, however [25]. Occasional hyperintensity within regenerative nodules has been attributed to other substances less clearly understood (e.g., protein, copper) [33,74]. On unenhanced T2weighted images, regenerative nodules are typically indistinct or mildly hypointense. Most regenerative nodules enhance to the same degree as the adjacent liver, or show slightly less enhancement. Uptake and excretion of hepatocellular agents are usually preserved and, as a result, all regenerative nodules have a similar appearance on the hepatocellular phase [25].

\subsubsection{Dysplastic Nodules}

Dysplastic nodules have variable appearances on MRI, and their signal intensities may overlap with those of regenerative nodules (low-grade dysplastic nodules) and well-differentiated HCC (high-grade dysplastic nodules). T1-weighted images are generally not helpful in the evaluation of dysplastic nodules due to the variable (low, intermediate, high) signal intensity of these lesions. On T2-weighted images, low-grade dysplastic nodules tend to have low signal intensity relative to adjacent liver, whereas that of high-grade dysplastic nodules tends to be slightly increased [25]. Both regenerative and dysplastic nodules may occasionally infarct, resulting in T2 signal hyperintensity on MRI, and mimic hypovascular HCC [75].

On contrast-enhanced MRI, low-grade dysplastic nodules are indistinguishable from regenerative nodules, whereas high-grade dysplastic nodules, which receive increaseing supply from the hepatic artery, may be indistinguishable from, and mistaken for, well-differentiated HCC [24, $29,34,76,77]$. Lesional enhancement during the arterial phase after contrast bolus administration is the imaging correlate to hypervascularity. This is considered an essential characteristic of HCC, and is used as the only radiologic feature on contrast-enhanced CT or MRI for imaging diagnosis prior to listing (UNOS) [78].

\subsubsection{Small Nodules and HCC Nodules}

On T1-weighted and T2-weighted images, small HCC may have variable signal intensities; moderately increased T2 signal is more specific for malignancy, since only a minority of (generally, infarcted) dysplastic or regen- 
erative nodules are hyperintense [75,79].

Approximately $80 \%$ - 90\% of HCC are hypervascular and avidly enhance during the arterial phase of dynamic contrast-enhanced CT or MRI. The remaining 10\% $20 \%$ are hypovascular malignancies and show less contrast enhancement on the arterial phase compared to the surrounding liver $[25,53,80]$. An enhancing tumor capsule on the delayed phase of contrast administration is evident in $65 \%-89 \%$ of large HCC, and though this finding is not required for diagnosis, its presence is highly specific (up to $96 \%$ ) $[24,25,42]$.

The complexity of liver imaging, including the limitations of the procedures currently available, emphasizes the need for a multidisciplinary approach to achieve the most accurate diagnosis and best possible treatment of cirrhosis-associated hepatocellular nodules.

\section{Summary}

The early detection of $\mathrm{HCC}$ in high-risk individuals is rather limited, with US and serum AFP currently being the most widely used tests. A major advancement in the diagnosis of HCC, however, is the development of LIRADS, which promises to improve the care of patients at risk for having $\mathrm{HCC}$ by reducing variability in lesion interpretation and reporting, thereby improving communication among providers, and facilitating not only decision-making for individual patient care but also standardization for quality assurance and research endeavors. Given recent major advancements in liver imaging, particularly using MRI, and given the optimal diagnosis of the difficult cirrhotic nodule requires a high degree of expertise among subspecialist hepatobiliary radiologists.

\section{REFERENCES}

[1] H. B. El-Serag, "Epidemiology of Viral Hepatitis and Hepatocellular Carcinoma," Gastroenterology, Vol. 142, No. 6, 2012, pp. 1264-1273.

[2] D. M. Parkin, F. Bray, J. Ferlay, et al., "Global Cancer Statistics, 2002," CA Cancer Journal for Clinicians, Vol. 55, No. 2, 2005, pp. 74-108. doi:10.3322/canjclin.55.2.74

[3] J. M. Llovet, A. Burroughs and J. Bruix, "Hepatocellular Carcinoma," Lancet, Vol. 362, No. 9399, 2003, pp. 19071917. doi:10.1016/S0140-6736(03)14964-1

[4] American Cancer Society, "Cancer Facts and Figures 2012," 2012.

http://www.cancer.org/acs/groups/content/@epidemiolog ysurveilance/documents/document/acspc-031941.pdf

[5] B. Q. Starley, C. J. Calcagno and S. A. Harrison, "NonAlcoholic Fatty Liver Disease and Hepatocellular Carcinoma: A Weighty Connection," Hepatology, Vol. 51, No. 5, 2010, pp. 1820-1832. doi:10.1002/hep.23594

[6] H. B. El-Serag, H. Hampel and F. Javadi, "The Association between Diabetes and Hepatocellular Carcinoma: A Systematic Review of Epidemiologic Evidence," Clinical
Gastroenterology and Hepatology, Vol. 4, No. 3, 2006, pp. 369-380. doi:10.1016/j.cgh.2005.12.007

[7] B. H. Zhang, B. H. Yang and Z. Y. Tang, "Randomized Controlled Trial of Screening for Hepatocellular Carcinoma," Journal of Cancer Research and Clinical Oncology, Vol. 130, No. 7, 2004, pp. 417-422.

doi:10.1007/s00432-004-0552-0

[8] R. Aghoram, P. Cai and J. A. Dickinson, "Alpha-Foetoprotein and/or Liver Ultrasonography for Screening of Hepatocellular Carcinoma in Patients with Chronic Hepatitis B," Cochrane Database Systematic Reviews, Vol. 9, 2012, Article ID: CD002799. doi:10.1002/14651858.CD002799.pub2

[9] J. C. Trinchet, C. Chaffaut, V. Bourcier, et al., "Ultrasonographic Surveillance of Hepatocellular Carcinoma in Cirrhosis: A Randomized Trial Comparing 3- and 6Month Periodicities," Hepatology, Vol. 54, No. 6, 2011, pp. 1987-1997. doi:10.1002/hep.24545

[10] L. Bolondi, S. Sofia, S. Siringo, et al., "Surveillance Programme of Cirrhotic Patients for Early Diagnosis and Treatment of Hepatocellular Carcinoma: A Cost Effectiveness Analysis," Gut, Vol. 48, No. 2, 2001, pp. 251259. doi:10.1136/gut.48.2.251

[11] G. Bertino, A. Ardiri, M. Malaguarnera, et al., "Hepatocellualar Carcinoma Serum Markers," Seminars in Oncology, Vol. 39, No. 4, 2012, pp. 410-433. doi:10.1053/j.seminoncol.2012.05.001

[12] R. Masuzaki, S. J. Karp and M. Omata, "New Serum Markers of Hepatocellular Carcinoma," Seminars in Oncology, Vol. 39, No. 4, 2012, pp. 434-439. doi:10.1053/j.seminoncol.2012.05.009

[13] A. S. Purysko, E. M. Remer, C. P. Coppa, et al., "LIRADS: A Case-Based Review of the New Categorization of Liver Findings in Patients with End-Stage Liver Disease," Radiographics, Vol. 32, No. 7, 2012, pp. 19771995. doi: $10.1148 / \mathrm{rg} .327125026$

[14] LI-RADS, "Liver Imaging-Reporting and Data System," 2013.

http://www.acr.org/Quality-Safety/Resources/LIRADS

[15] D. J. Becker-Weidman, B. Kalb, P. Sharma, et al., "Hepatocellular Carcinoma Lesion Characterization: Single-Institution Clinical Performance Review of Multiphase Gadolinium-Enhanced MR Imaging - Comparison to Prior Same-Center Results after MR Systems Improvements," Radiology, Vol. 261, No. 3, 2011, pp. 824-833. doi:10.1148/radiol.11110157

[16] P. Sharma, B. Kalb, H. D. Kitajima, et al., "Optimization of Single Injection Liver Arterial Phase Gadolinium Enhanced MRI Using Bolus Track Real-Time Imaging," Journal of Magnetic Resonance Imaging, Vol. 33, No. 1, 2011, pp. 110-118. doi:10.1002/jmri.22200

[17] S. C. Cunningham and S. Farooqui, "Subspecialty Radiology and Surgery," Hepatobiliary \& Pancreatic Diseases International, Vol. 12, No. 2, 2013, pp. 122-124.

[18] S. F. Altekruse, K. A. McGlynn, L. A. Dickie, et al., "Hepatocellular Carcinoma Confirmation, Treatment, and Survival in Surveillance, Epidemiology, and End Results Registries, 1992-2008," Hepatology, Vol. 55, No. 2, 2012, pp. 476-482. doi:10.1002/hep. 24710 
[19] National Comprehensive Cancer Network, "NCCN Clinical Practice Guidelines in Oncology," 2013. http://www.nccn.org/professionals/physician_gls/pdf/hep atobiliary.pdf

[20] D. N. Hoffer, A. Finelli, R. Chow, et al., "Structured Electronic Operative Reporting: Comparison with Dictation in Kidney Cancer Surgery," International Journal of Medical Informatics, Vol. 81, No. 3, 2012, pp. 182-191. doi:10.1016/j.ijmedinf.2011.11.008

[21] D. A. Cowan, M. B. Sands, S. M. Rabizadeh, et al., "Electronic Templates versus Dictation for the Completion of Mohs Micrographic Surgery Operative Notes," Dermatologic Surgery, Vol. 33, No. 5, 2007, pp. 588-595. doi:10.1111/j.1524-4725.2007.33120.x

[22] L. A. Mack, K. Dabbs and W. J. Temple, "Synoptic Operative Record for Point of Care Outcomes: A Leap Forward in Knowledge Translation," European Journal of Surgical Oncology, Vol. 36, Suppl. 1, 2010, pp. S44-49.

[23] J. M. Lee, F. Trevisani, V. Vilgrain, et al., "Imaging Diagnosis and Staging of Hepatocellular Carcinoma," Liver Transplantation, Vol. 17, Suppl. 2, 2011, pp. S34-43.

[24] International Working Party, "Terminology of Nodular Hepatocellular Lesions," Hepatology, Vol. 22, No. 3, 1995, pp. 983-993.

[25] R. F. Hanna, D. A. Aguirre, N. Kased, et al., "CirrhosisAssociated Hepatocellular Nodules: Correlation of Histopathologic and MR Imaging Features1," Radiographics, Vol. 28, No. 3, 2008, pp. 747-769. doi:10.1148/rg.283055108

[26] G. Brancatelli, M. P. Federle, L. Grazioli, et al., "Large Regenerative Nodules in Budd-Chiari Syndrome and Other Vascular Disorders of the Liver," American Journal of Roentgenology, Vol. 178, No. 4, 2002, pp. 877-883. doi:10.2214/ajr.178.4.1780877

[27] A. Qayyum, A. Graser, A. Westphalen, et al., "CT of Benign Hypervascular Liver Nodules in Autoimmune Hepatitis," American Journal of Roentgenology, Vol. 183, No. 6, 2004, pp. 1573-1576. doi:10.2214/ajr.183.6.01831573

[28] G. A. Krinsky, V. S. Lee, M. T. Nguyen, et al., "Siderotic Nodules at MR Imaging: Regenerative or Dysplastic?" Journal of Computer Assisted Tomography, Vol. 24, No. 5, 2000, pp. 773-776.

[29] G. A. Krinsky, V. S. Lee, N. D. Theise, et al., "Hepatocellular Carcinoma and Dysplastic Nodules in Patients with Cirrhosis: Prospective Diagnosis with MR Imaging and Explantation Correlation1," Radiology, Vol. 219, No. 2, 2001, pp. 445-454.

[30] N. D. Theise, M. Schwartz, C. Miller, et al., "Macroregenerative Nodules and Hepatocellular Carcinoma in FortyFour Sequential Adult Liver Explants with Cirrhosis," Hepatology, Vol. 16, No. 4, 1992, pp. 949-955. doi:10.1002/hep.1840160416

[31] S. Efremidis and P. Hytiroglou, "The Multistep Process of Hepatocarcinogenesis in Cirrhosis with Imaging Correlation," European Radiology, Vol. 12, No. 4, 2002, pp. 753-764. doi:10.1007/s00330-001-1142-z

[32] M. Borzio, S. Fargion, F. Borzio, et al., "Impact of Large Regenerative, Low Grade and High Grade Dysplastic No- dules in Hepatocellular Carcinoma Development," Journal of Hepatology, Vol. 39, No. 2, 2003, pp. 208-214. doi:10.1016/S0168-8278(03)00190-9

[33] J. M. Willatt, H. K. Hussain, S. Adusumilli, et al., "MR Imaging of Hepatocellular Carcinoma in the Cirrhotic Liver: Challenges and Controversies1," Radiology, Vol. 247, No. 2, 2008, pp. 311-330. doi:10.1148/radiol.2472061331

[34] M. Hayashi, O. Matsui, K. Ueda, et al., "Correlation between the Blood Supply and Grade of Malignancy of Hepatocellular Nodules Associated with Liver Cirrhosis: Evaluation by CT during Intraarterial Injection of Contrast Medium," American Journal of Roentgenology, Vol. 172, No. 4, 1999, pp. 969-976. doi:10.2214/ajr.172.4.10587130

[35] J. Bruix and M. Sherman, "Management of Hepatocellular Carcinoma: An Update," Hepatology, Vol. 53, No. 3, 2011, pp. 1020-1022. doi:10.1002/hep.24199

[36] L. Di Tommaso, G. Franchi, Y. N. Park, et al., "Diagnostic Value of HSP70, Glypican 3, and Glutamine Synthetase in Hepatocellular Nodules in Cirrhosis," Hepatology, Vol. 45, No. 3, 2007, pp. 725-734. doi:10.1002/hep. 21531

[37] International Consensus Group for Hepatocellular Neoplasia, "Pathologic Diagnosis of Early Hepatocellular Carcinoma: A Report of the International Consensus Group for Hepatocellular Neoplasia," Hepatology, Vol. 49, No. 2, 2009, pp. 658-664. doi:10.1002/hep.22709

[38] M. Roncalli, M. Borzio and L. Di Tommaso, "Hepatocellular Dysplastic Nodules," Hepatology Research, Vol. 37, Suppl. S2, 2007, pp. S125-S134.

[39] M. Kojiro and T. Roskams, "Early Hepatocellular Carcinoma and Dysplastic Nodules," Seminars in Liver Disease, Vol. 25, No. 2, 2005, pp. 133-142.

[40] O. Matsui, M. Kadoya, T. Kameyama, et al., "Benign and Malignant Nodules in Cirrhotic Livers: Distinction Based on Blood Supply," Radiology, Vol. 178, No. 2, 1991, pp. 493-497.

[41] A. E. Holland, E. M. Hecht, W. Y. Hahn, et al., "Importance of Small $(<$ or $=20-\mathrm{mm})$ Enhancing Lesions Seen Only during the Hepatic Arterial Phase at MR Imaging of the Cirrhotic Liver: Evaluation and Comparison with Whole Explanted Liver," Radiology, Vol. 237, No. 3, 2005, pp. 938-944. doi:10.1148/radiol.2373041364

[42] J. A. Marrero, H. K. Hussain, H. V. Nghiem, et al., "Improving the Prediction of Hepatocellular Carcinoma in Cirrhotic Patients with an Arterially-Enhancing Liver Mass," Liver Transplantation, Vol. 11, No. 3, 2005, pp. 281-289. doi:10.1002/lt.20357

[43] J. S. Yu, K. W. Kim, K. B. Sung, et al., "Small Arterial-Portal Venous Shunts: A Cause of Pseudolesions at Hepatic Imaging," Radiology, Vol. 203, No. 3, 1997, pp. 737-742.

[44] J.-S. Yu, K. W. Kim, M.-G. Jeong, et al., "Nontumorous Hepatic Arterial-Portal Venous Shunts: MR Imaging Findings 1," Radiology, Vol. 217, No. 3, 2000, pp. 750756.

[45] G. D. Dodd, R. L. Baron, J. H. Oliver, et al., "Spectrum 
of Imaging Findings of the Liver in End-Stage Cirrhosis: Part I, Gross Morphology and Diffuse Abnormalities," American Journal of Roentgenology, Vol. 173, No. 4, 1999, pp. 1031-1036. doi:10.2214/ajr.173.4.10511173

[46] A. Colli, M. Fraquelli, G. Casazza, et al., "Accuracy of Ultrasonography, Spiral CT, Magnetic Resonance, and Alpha-Fetoprotein in Diagnosing Hepatocellular Carcinoma: A Systematic Review," The American Journal of Gastroenterol, Vol. 101, No. 3, 2006, pp. 513-523. doi:10.1111/j.1572-0241.2006.00467.x

[47] V. de Ledinghen, D. Laharie, R. Lecesne, et al., "Detection of Nodules in Liver Cirrhosis: Spiral Computed Tomography or Magnetic Resonance Imaging? A Prospective Study of 88 Nodules in 34 Patients," European Journal of Gastroenterology \& Hepatology, Vol. 14, No. 2, 2002, pp. 159-165. doi:10.1097/00042737-200202000-00010

[48] A. Rode, B. Bancel, P. Douek, et al., "Small Nodule Detection in Cirrhotic Livers: Evaluation with US, Spiral CT, and MRI and Correlation with Pathologic Examination of Explanted Liver," Journal of Computer Assisted Tomography, Vol. 25, No. 3, 2001, pp. 327-336. doi:10.1097/00004728-200105000-00001

[49] G. L. Bennett, G. A. Krinsky, R. J. Abitbol, et al., "Sonographic Detection of Hepatocellular Carcinoma and Dysplastic Nodules in Cirrhosis: Correlation of Pretransplantation Sonography and Liver Explant Pathology in 200 Patients," AJR American Journal of Roentgenology, Vol. 179, No. 1, 2002, pp. 75-80. doi:10.2214/ajr.179.1.1790075

[50] A. Shimizu, K. Ito, S. Koike, et al., "Cirrhosis or Chronic Hepatitis: Evaluation of Small $(<$ or $=2-\mathrm{cm})$ Early-Enhancing Hepatic Lesions with Serial Contrast-Enhanced Dynamic MR Imaging," Radiology, Vol. 226, No. 2, 2003, pp. 550-555. doi:10.1148/radiol.2262011967

[51] Y. Y. Jeong, D. G. Mitchell and T. Kamishima, "Small $(<20 \mathrm{~mm})$ Enhancing Hepatic Nodules Seen on Arterial Phase MR Imaging of the Cirrhotic Liver: Clinical Implications," AJR American Journal of Roentgenology, Vol. 178, No. 6, 2002, pp. 1327-1334. doi:10.2214/ajr.178.6.1781327

[52] P. Hytiroglou and N. Theise, "Differential Diagnosis of Hepatocellular Nodular Lesions," Seminars in Diagnostic Pathology, Vol. 15, No. 4, 1998, pp. 285-299.

[53] L. Bolondi, S. Gaiani, N. Celli, et al., "Characterization of Small Nodules in Cirrhosis by Assessment of Vascularity: The Problem of Hypovascular Hepatocellular Carcinoma," Hepatology, Vol. 42, No. 1, 2005, pp. 27-34. doi:10.1002/hep. 20728

[54] T. C. Lauenstein, K. Salman, R. Morreira, et al., "Gadolinium-Enhanced MRI for Tumor Surveillance Before Liver Transplantation: Center-Based Experience," American Journal of Roentgenology, Vol. 189, No. 3, 2007, pp. 663- 670. doi:10.2214/AJR.07.2171

[55] C. J. R. Stewart, J. Coldewey and I. S. Stewart, "Comparison of Fine Needle Aspiration Cytology and Needle Core Biopsy in the Diagnosis of Radiologically Detected Abdominal Lesions," Journal of Clinical Pathology, Vol. 55, No. 2, 2002, pp. 93-97. doi:10.1136/jep.55.2.93
[56] F. O. Durand, J. M. Regimbeau, J. Belghiti, et al., "Assessment of the Benefits and Risks of Percutaneous Biopsy before Surgical Resection of Hepatocellular Carcinoma," Journal of Hepatology, Vol. 35, No. 2, 2001, pp. 254-258. doi:10.1016/S0168-8278(01)00108-8

[57] J. H. Lim, D. Choi, S. H. Kim, et al., "Detection of Hepatocellular Carcinoma: Value of Adding Delayed Phase Imaging to Dual-Phase Helical CT," American Journal of Roentgenology, Vol. 179, No. 1, 2002, pp. 67-73. doi:10.2214/ajr.179.1.1790067

[58] R. C. Carlos, H. M. Kim, H. K. Hussain, et al., "Developing a Prediction Rule to Assess Hepatic Malignancy in Patients with Cirrhosis," American Journal of Roentgenology, Vol. 180, No. 4, 2003, pp. 893-900. doi:10.2214/ajr.180.4.1800893

[59] R. Iannaccone, A. Laghi, C. Catalano, et al., "Hepatocellular Carcinoma: Role of Unenhanced and Delayed Phase Multi-Detector Row Helical CT in Patients with Cirrhosis," Radiology, Vol. 234, No. 2, 2005, pp. 460-467. doi:10.1148/radiol.2342031202

[60] D. Choi, D. G. Mitchell, S. K. Verma, et al., "Hepatocellular Carcinoma with Indeterminate or False-Negative Findings at Initial MR Imaging: Effect on Eligibility for Curative Treatment: Initial Observations," Radiology, Vol. 244, No. 3, 2007, pp. 776-783. doi:10.1148/radiol.2443061355

[61] J.-J. Chung, J. S. Yu, J. H. Kim, et al., "Nonhypervascular Hypoattenuating Nodules Depicted on Either Portal or Equilibrium Phase Multiphasic CT Images in the Cirrhotic Liver," American Journal of Roentgenology, Vol. 191, No. 1, 2008, pp. 207-214. doi:10.2214/AJR.07.3409

[62] A. E. Holland, E. M. Hecht, W. Y. Hahn, et al., "Importance of Small $(\leq 20-\mathrm{mm})$ Enhancing Lesions Seen Only during the Hepatic Arterial Phase at MR Imaging of the Cirrhotic Liver: Evaluation and Comparison with Whole Explanted Liver," Radiology, Vol. 237, No. 3, 2005, pp. 938-944. doi:10.1148/radiol.2373041364

[63] S. H. Choi, J. M. Lee, N. C. Yu, et al., "Hepatocellular Carcinoma in Liver Transplantation Candidates: Detection with Gadobenate Dimeglumine-Enhanced MRI," American Journal of Roentgenology, Vol. 191, No. 2, 2008, pp. 529-536. doi:10.2214/AJR.07.2565

[64] R. B. Freeman, A. Mithoefer, R. Ruthazer, et al., "Optimizing Staging for Hepatocellular Carcinoma before Liver Transplantation: A Retrospective Analysis of the UNOS/OPTN Database," Liver Transplantation, Vol. 12, No. 10, 2006, pp. 1504-1511. doi:10.1002/lt.20847

[65] A. Forner, R. N. Vilana, C. Ayuso, et al., "Diagnosis of Hepatic Nodules $20 \mathrm{~mm}$ or Smaller in Cirrhosis: Prospective Validation of the Noninvasive Diagnostic Criteria for Hepatocellular Carcinoma," Hepatology, Vol. 47, No. 1, 2008, pp. 97-104. doi:10.1002/hep.21966

[66] B. Taouli, V. R. Vilgrain, E. Dumont, et al., "Evaluation of Liver Diffusion Isotropy and Characterization of Focal Hepatic Lesions with Two Single-Shot Echo-Planar MR Imaging Sequences: Prospective Study in 66 Patients," Radiology, Vol. 226, No. 1, 2003, pp. 71-78. doi:10.1148/radiol.2261011904

[67] T. Parikh, S. J. Drew, V. S. Lee, et al., "Focal Liver Lesion 
Detection and Characterization with Diffusion-Weighted MR Imaging: Comparison with Standard Breath-Hold T2-Weighted Imaging," Radiology, Vol. 246, No. 3, 2008, pp. 812-822. doi:10.1148/radiol.2463070432

[68] J. H. Lim, D. Choi, S. K. Cho, et al., "Conspicuity of Hepatocellular Nodular Lesions in Cirrhotic Livers at Ferumoxides-Enhanced MR Imaging: Importance of Kupffer Cell Number," Radiology, Vol. 220, No. 3, 2001, pp. 669-676. doi:10.1148/radiol.2203001777

[69] K. I. Ringe, D. B. Husarik, C. B. Sirlin, et al., "Gadoxetate Disodium-Enhanced MRI of the Liver: Part 1, Protocol Optimization and Lesion Appearance in the Noncirrhotic Liver," American Journal of Roentgenology, Vol. 195, No. 1, 2010, pp. 13-28. doi:10.2214/AJR.10.4392

[70] D. Marin, M. Di Martino, A. Guerrisi, et al., "Hepatocellular Carcinoma in Patients with Cirrhosis: Qualitative Comparison of Gadobenate Dimeglumine-Enhanced MR Imaging and Multiphasic 64-Section CT1," Radiology, Vol. 251, No. 1, 2009, pp. 85-95. doi:10.1148/radiol.2511080400

[71] I. Cruite, M. Schroeder, E. M. Merkle, et al., "Gadoxetate Disodium-Enhanced MRI of the Liver: Part 2, Protocol Optimization and Lesion Appearance in the Cirrhotic Liver," American Journal of Roentgenology, Vol. 195, No. 1, 2010, pp. 29-41. doi:10.2214/AJR.10.4538

[72] G. Brancatelli, R. L. Baron, M. S. Peterson, et al., "Helical CT Screening for Hepatocellular Carcinoma in Patients with Cirrhosis: Frequency and Causes of FalsePositive Interpretation," American Journal of Roentgenology, Vol. 180, No. 4, 2003, pp. 1007-1014. doi:10.2214/ajr.180.4.1801007

[73] M. Kudo and T. Okanoue, "Management of Hepatocellular Carcinoma in Japan: Consensus-Based Clinical Practice Manual Proposed by the Japan Society of Hepatol- ogy," Oncology, Vol. 72, Suppl. 1, 2007, pp. 2-15.

[74] G. A. Krinsky and G. Israel, "Nondysplastic Nodules That Are Hyperintense on T1-Weighted Gradient-Echo MR Imaging: Frequency in Cirrhotic Patients Undergoing Transplantation," American Journal of Roentgenology, Vol. 180, No. 4, 2003, pp. 1023-1027. doi:10.2214/ajr.180.4.1801023

[75] T. Kim, R. L. Baron and M. A. Nalesnik, "Infarcted Regenerative Nodules in Cirrhosis," American Journal of Roentgenology, Vol. 175, No. 4, 2000, pp. 1121-1125. doi:10.2214/ajr.175.4.1751121

[76] B. Choi, J. Han, S. Hong, et al., "Dysplastic Nodules of the Liver: Imaging Findings," Abdominal Imaging, Vol. 24, No. 3, 1999, pp. 250-257. doi: $10.1007 / \mathrm{s} 002619900490$

[77] G. A. Krinsky, N. D. Theise, N. M. Rofsky, et al., "Dysplastic Nodules in Cirrhotic Liver: Arterial Phase Enhancement at CT and MR Imaging-A Case Report," Radiology, Vol. 209, No. 2, 1998, pp. 461-464.

[78] N. L. Kelekis, R. C. Semelka, S. Worawattanakul, et al., "Hepatocellular Carcinoma in North America: A Multiinstitutional Study of Appearance on T1-Weighted, T2Weighted, and Serial Gadolinium-Enhanced Gradient-Echo Images," American Journal of Roentgenology, Vol. 170, No. 4, 1998, pp. 1005-1013. doi:10.2214/ajr.170.4.9530051

[79] J. P. Earls, N. D. Theise, J. C. Weinreb, et al., "Dysplastic Nodules and Hepatocellular Carcinoma: Thin-Section MR Imaging of Explanted Cirrhotic Livers with Pathologic Correlation," Radiology, Vol. 201, No. 1, 1996, pp. 207214.

[80] M. Sherman, "Diagnosis of Small Hepatocellular Carcinoma," Hepatology, Vol. 42, No. 1, 2005, pp. 14-16. doi:10.1002/hep.20790 